\title{
A novel matrix approach for controlling the invariant densities of chaotic maps
}

\author{
Alan Rogers ${ }^{\mathrm{a}, *}$, Robert Shorten ${ }^{\mathrm{b}}$, Daniel M. Heffernan ${ }^{\mathrm{c}}$ \\ a Department of Electronic Engineering, NUI Maynooth, Maynooth, Co. Kildare, Ireland \\ ${ }^{\mathrm{b}}$ Hamilton Institute, NUI Maynooth, Maynooth, Co. Kildare, Ireland \\ ${ }^{\mathrm{c}}$ Department of Mathematical Physics, NUI Maynooth, Maynooth, Co. Kildare, Ireland
}

Accepted 11 May 2006

Communicated by Prof. M.S. El Naschie

\begin{abstract}
Recent work on positive matrices has resulted in a new matrix method for generating chaotic maps with arbitrary piecewise constant invariant densities, sometimes known as the inverse Frobenius-Perron problem (IFPP). In this paper, we give an extensive introduction to the IFPP, describing existing methods for solving it, and we describe our new matrix approach for solving the IFPP.
\end{abstract}

(C) 2006 Elsevier Ltd. All rights reserved.

\section{Introduction}

The inverse Frobenius-Perron problem (IFPP) refers to the problem of creating chaotic maps with desired invariant densities [1]. A number of different approaches to the problem have appeared in the literature, including conjugate-function, control-based, and matrix-based approaches [2-4]. The IFPP can be seen as a stepping-stone to new applications of chaos, which will only burgeon when systematic synthesis and control techniques are available. Some researchers, unaware of the work on the IFPP, tend to use the logistic map for every conceivable application. We do not feel that this is the best approach when there are a variety of ways available to control and synthesize maps with desired properties.

We recently developed a straightforward matrix approach to the IFPP which is completely mechanical, requiring little effort to generate a chaotic map with a desired invariant density. This work has potential applications in data modelling and creating random numbers with desired statistics, and was first described in [5]. In this paper, after an extensive introduction to the IFPP, we look in more detail at our matrix method, especially controlling the overall dynamics of system, and the effect of the various parameters on Lyapunov exponent and mixing rates. We also examine what happens to invariant densities when we switch between chaotic maps.

\footnotetext{
${ }^{*}$ Corresponding author. Tel.: +35317086067; fax: +35317086027.

E-mail addresses: alan.rogers@eeng.nuim.ie, alan.rogers@eeng.may.ie (A. Rogers).
} 


\section{Background to the inverse Frobenius-Perron problem}

Normally, when we iterate a chaotic map starting from some initial condition $x_{0}$, the iterates fall chaotically on some attractor. It can be difficult to see the attractor if we just look at the time-series of the iterates. If we partition the state space into a series of bins, and count the fraction of iterates in each bin, a statistical picture of the chaotic attractor emerges. For almost all initial conditions, the same picture emerges: a unique invariant density $\rho(x)$. It is true that for certain initial conditions (rational numbers, or extreme points) other invariant densities are possible, but for the maps we will be considering, there will be only one physically relevant invariant density [6]. This density is stable if a small amount of noise is added to the system. In order to characterize the density mathematically, we consider an ensemble of initial conditions described by a probability density function $\rho_{0}(x)$ and observe how it changes as the entire ensemble is iterated. Eventually, the invariant density is reached, after, say $n$ iterates. Further iteration of the ensemble of points just gives the invariant density $\rho(x)$ each time. The collection of initial conditions has reached a stable fixedpoint of the Frobenius-Perron equation where the Frobenius-Perron operator (FPO), $P$, is a linear operator acting on distributions of points [1]:

$$
P \rho(x)=\frac{\mathrm{d}}{\mathrm{d} x} \int_{f^{-1}([a, x])} \rho(y) \mathrm{d} y .
$$

For piecewise linear maps, Eq. (1) can be rewritten in the following more convenient form:

$$
P \rho(y)=\sum_{x \in f^{-1}(y)} \frac{\rho(x)}{\left|f^{\prime}(x)\right|} .
$$

Solving Eq. (1) is a difficult (if not impossible) problem for arbitrary continuous maps: Firstly, the invariant density may be a fractal or Cantor set, in which case the intervals concerned have measure zero; secondly, it may not be possible to solve the resulting functional equations, assuming the invariant measure is continuous. So the inverse problem, of choosing an arbitrary invariant measure, and finding which map gives rise to it, must seem like quite an impossible task. All is not lost though. There are two main approaches to the IFPP in the literature. The first method uses a conjugate function approach, the second is based on approximation of the FPO by a Markov matrix.

The conjugate function approach, which was first described by Grossman and Thomae [2], makes use of the following equivalence relation between two mappings: The maps $f: I \rightarrow I$ and $g: J \rightarrow J$ on intervals $I$ and $J$ are conjugate if there exists a one-to-one map $h: I \stackrel{\text { onto }}{\rightarrow} J$ such that

$$
g(x)=h\left(f\left[h^{-1}(x)\right]\right) .
$$

The conjugating function $h$, assumed to be continuous and sufficiently smooth, establishes a one-to-one correspondence between the iterates of the two maps $f$ and $g$. The invariant densities of $g$ and $h$ are related as follows:

$$
\rho_{g}(x)=\rho_{f}\left[h^{-1}(x)\right]\left|\frac{\mathrm{d} h^{-1}(x)}{\mathrm{d} x}\right| .
$$

Numerous examples are given in the paper by Grossman. Also, this approach can be used to find the invariant density of the logistic map. It is conjugate to the tent map through the conjugating function $h(x)=\sin ^{2}\left(\frac{\pi x}{2}\right)$ when $r=4$ (see [7] for details). The invariant density of the tent map is constant and equals one, and so Eq. (4) reduces to

$$
\rho_{g}(x)=\left|\frac{\mathrm{d} h^{-1}(x)}{\mathrm{d} x}\right|
$$

The inverse function $h^{-1}$ can be shown to be

$$
h^{-1}(x)=\frac{2}{\pi} \sin ^{-1} \sqrt{x}
$$

We can find the derivative, and thus the required invariant density, as follows:

$$
\rho_{g}(x)=\frac{\mathrm{d}}{\mathrm{d} x}\left(\frac{2}{\pi} \sin ^{-1} \sqrt{x}\right)=\frac{1}{\pi \sqrt{x(1-x)}} .
$$

Fig. 1 illustrates the two conjugate maps, the conjugating function $h(x)=\sin ^{2}\left(\frac{\pi x}{2}\right)$, and the invariant densities of the logistic and tent maps.

The Markov matrix approach, upon which our new results are based, was first suggested by Ulam [8]. The FPO is a Markov operator, in the sense that the density at step $n+1$ is only a function of the density at step $n$ (see [9]). Ulam 

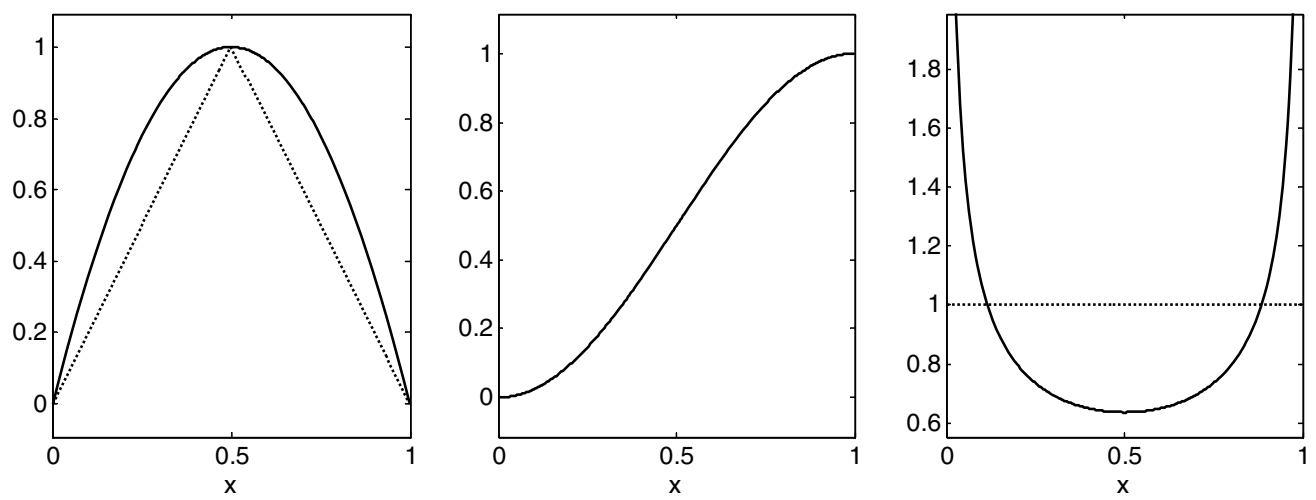

Fig. 1. (i) Logistic and tent maps, (ii) conjugating function, (iii) invariant densities of both maps.

suggested that the state-space (the unit interval in all of our work) be arbitrarily partitioned into $N$ subintervals, $I_{1}, \ldots, I_{N}$. Then define a probability vector at step $n$ :

$$
P_{n}=\left\{\int_{I_{1}} \rho_{n}(x) \mathrm{d} x, \ldots, \int_{I_{N}} \rho_{n}(x) \mathrm{d} x\right\} .
$$

Now introduce an $N \times N$ transition matrix, $W$, which gives the probabilities of iterates moving from any subinterval to any other subinterval in the partition. Ulam hypothesized that the FPO could now be approximated by the following matrix equation:

$$
P_{n+1}=W P_{n} \text {. }
$$

It is clear that as $N \rightarrow \infty$, Eq. (9) gives a better and better approximation of the FPO. Ulam's hypothesis was later proved by $\mathrm{Li}$ [10]. It is remarkable that the statistical properties of chaotic systems can be represented by such a simple linear equation, allowing us to bring many of the results of positive matrix theory to bear on the problem.

There has been a large amount of work done on controlling the statistical properties of 1-D maps in recent years. Apart from the seminal work of Grossman and Thomae cited earlier, we mention the paper of Baranovsky and Daems [11], in which piecewise linear Markov maps are used as references whose statistical properties are known. These maps are then transformed into non-Markov maps and smooth maps, using conjugating functions. They also consider the problem of designing maps with prescribed correlation functions. Pingel and co-workers [12] manage to solve the Frobenius-Perron equation exactly for a class of unimodal chaotic maps, whose invariant densities are members of a class of beta distributions. The group led by Setti have studied the Markov approach to the IFPP with a view to applying it to signal processing tasks (see especially [13] and the copious references therein). They consider a variety of piecewise linear maps including $n$-way Bernoulli shifts and develop a matrix-tensor formulation for quantifying high-order correlations of such maps. Another active area of research is the use of chaotic maps to model packet traffic in computer networks. Packet traffic is notoriously bursty, and chaotic maps are ideal for modelling the fractal properties of the traffic. Mondragon neatly summarizes the previous work in this area in [14], and introduces some different types of intermittency maps along with a discussion of the statistical properties of these maps.

\section{A matrix method for solving the IFPP}

A recent analysis of the transmission control protocol (TCP) in synchronised communication networks $[15,16]$ gave rise to a positive matrix with special properties that allows us to solve the IFPP in a novel and elegant way. In the TCP protocol, each data source is allocated a congestion window, which governs how much data it can send. The protocol uses an additive-increase multiplicative-decrease (AIMD) algorithm for allocating window size: if the network is uncongested, the amount of data each source can send increases additively, but when congestion is detected, the sources back off in a multiplicative way. It can be shown that if there are $n$ sources competing for some finite bandwidth, and all these sources are operating the TCP congestion control algorithm in the presence of a drop-tail buffer bottleneck, then the dynamics of the system may be modelled by the following matrix equation:

$$
W(k+1)=A W(k),
$$


where $W(k)$ is a vector of the congestion windows of each source. The matrix A has the form:

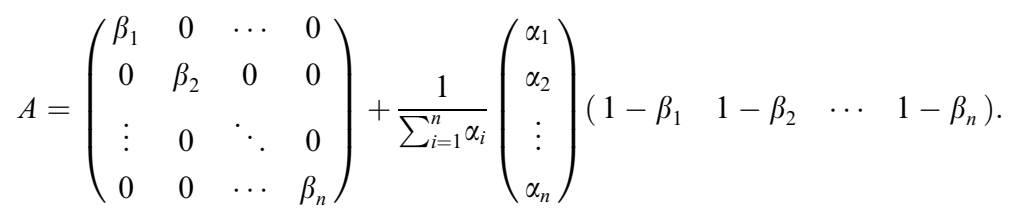

The $\alpha_{i}$ are the additive increase parameters for each of the $n$ sources, and the $\beta_{i}$ are the corresponding multiplicative decrease parameters. The matrix $A$ has many interesting properties, which we outline here:

1. Matrix $A$ is column stochastic (which means each column sums to 1).

2. The matrix is a positive matrix (all entries are positive real numbers).

3. The matrix has a single dominant eigenvalue of value 1 .

4. There is a single eigenvector of $A$ in the positive orthant called the Perron eigenvector, corresponding to the dominant eigenvalue, whose value is given by

$$
x_{\mathrm{P}}^{\mathrm{T}}=\left[\frac{\alpha_{1}}{1-\beta_{1}}, \frac{\alpha_{2}}{1-\beta_{2}}, \ldots, \frac{\alpha_{n}}{1-\beta_{n}}\right] .
$$

5. If the eigenvalues $\left(\lambda_{i}\right)$ and the $\beta_{i}$ are arranged in decreasing order, then the following interlacing scheme holds:

$$
1=\lambda_{1}>\beta_{1} \geqslant \lambda_{2} \geqslant \beta_{2} \geqslant \ldots \geqslant \lambda_{n} \geqslant \beta_{n} \text {. }
$$

The interlacing result and the form of the Perron eigenvector are given in the paper by Wirth [17], and are based on standard results on the symmetric eigenvalue problem (see, e.g., [18] or [19]).

We will be using the $A$ matrix to describe the transition probabilities between intervals in a partition. At the heart of our synthesis approach is the Perron eigenvector of $A$ : that it is parameterized in terms of the $\alpha_{i}$ and $\beta_{i}$ unlocks the inverse Frobenius-Perron problem.

Ulam's conjecture was that the principle eigenvector of a Markov process is the invariant density of that process, and that transformations on the interval could be approximated using this matrix approach.

We have a way of choosing our invariant density first, and automatically determining the Markov process that gave rise to it. The Markov process can then easily be turned into a 1-D map.

\subsection{Synthesis procedure}

Suppose that the desired invariant density (Perron eigenvector) $x_{\mathrm{d}}$ is

$$
x_{\mathrm{d}}^{\mathrm{T}}=\left[\delta_{1}, \delta_{2}, \ldots, \delta_{n}\right] .
$$

Choose the $\beta_{i}$ subject to the constraint: $0<\beta_{i}<1$. (The $\beta_{i}$ control how rapidly the map converges on the invariant density.) Often, we find it convenient to keep all the $\beta_{i}$ equal. Having chosen the $\beta_{i}$, determine the $\alpha_{i}$ as follows:

$$
\begin{aligned}
& \alpha_{1}=\delta_{1}\left(1-\beta_{1}\right), \\
& \alpha_{2}=\delta_{2}\left(1-\beta_{2}\right), \\
& \vdots \\
& \alpha_{n}=\delta_{n}\left(1-\beta_{n}\right) .
\end{aligned}
$$

Now form the matrix $A$ from the $\alpha_{i}$ and $\beta_{i}$ :

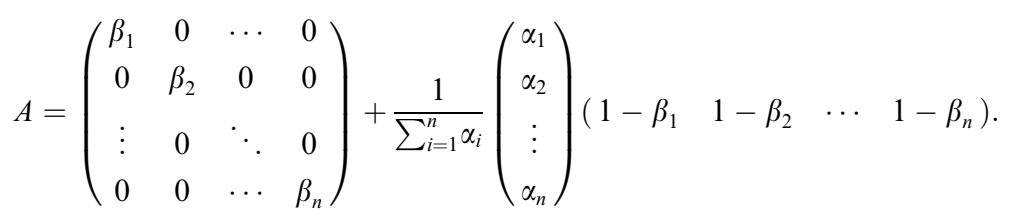

Next, we let the $A$ matrix represent a 1-D map on the unit interval to itself. We partition the unit interval into $n$ equal subintervals, $\left\{I_{1}, \ldots, I_{n}\right\}$ (assuming $A$ is an $n \times n$ matrix). Note that the partition can also be nonuniform (to be illustrated later). Let entry $a_{j i}$ of $A$ denote the probability of a transition from subinterval $I_{i}$ to $I_{j}$. (The order of the subscripts is 


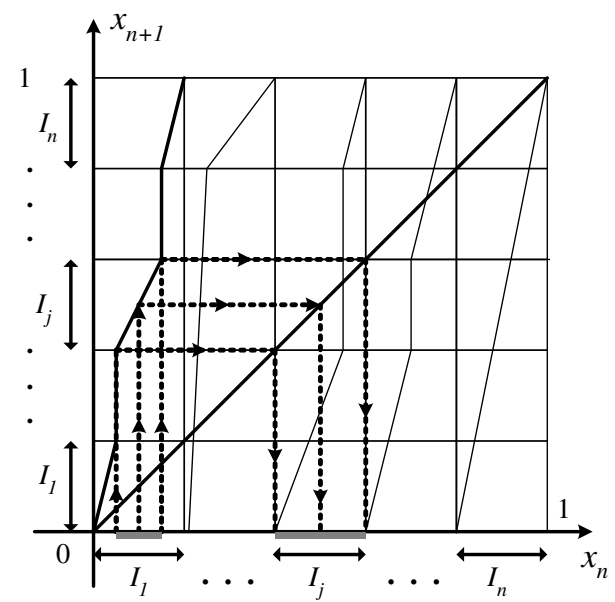

Fig. 2. Illustration of the construction of a 1-D map from a Markov matrix.

important here.) To construct the map, place a line segment of slope $\pm 1 / a_{j i}$ in the square defined by the subintervals $I_{i}, I_{j}$, as illustrated in Fig. 2. By controlling the slope of the line segment, we can control how much of the overall subinterval will interact with that portion of the map, which in turn relates to the transition probabilities. The most straightforward way of constructing the map is to start at the origin, and add the line segments end to end. In the figure, it can be seen how the probability of a transition from $I_{1}$ to $I_{j}$ is 0.5 , corresponding to a line of slope 2 in that region. In other words, $50 \%$ of the points in $I_{1}$ are mapped to $I_{j}$. The slope could have been -2 , and it could have been positioned anywhere within the square defined by those subintervals. This would affect the actual trajectory of the chaotic iterates, but it would not change the invariant density. A positive slope maps the extremities of an interval to the corresponding extremity (e.g., $\left.\inf \left(I_{i}\right) \rightarrow \inf \left(I_{j}\right)\right)$, while a negative slope maps each extremity to its opposite (e.g., $\left.\inf \left(I_{i}\right) \rightarrow \sup \left(I_{j}\right)\right)$.

\subsection{Examples}

We now give a mixture of examples of the synthesis procedure in action, using simple MATLAB code. The values of the $\beta_{i}$ are the same in each example.

(a) Invariant density $x_{\mathrm{d}}=[1,2,3]$. We let all the $\beta_{i}=0.1$ for convenience. The values of $\alpha_{i}$ are computed to be $[0.9,1.8,2.7]$. The transition matrix $A$ is then found to be:

$$
A=\left(\begin{array}{ccc}
0.25 & 0.15 & 0.15 \\
0.3 & 0.4 & 0.3 \\
0.45 & 0.45 & 0.55
\end{array}\right),
$$

$A$ is clearly column stochastic, and has eigenvalues of $[1,0.1,0.1]$, which we could have deduced from the interlacing property mentioned earlier. Fig. 3 shows the one-dimensional map corresponding to matrix $A$ and constructed in the manner outlined above. Fig. 4 is the invariant density of the map after 20000 iterations. The $y$-axis has been scaled to allow ready comparison with $x_{\mathrm{d}}$. A typical chaotic time-series from the map is shown in Fig. 5.

(b) The invariant density of the synthesized map has the shape of a sine-wave, but with an offset so that the values are all positive (see Figs. 6-8). The unit-interval is partitioned into 13 subintervals, giving a $13 \times 13$ transition matrix.

(c) In this example, we illustrate how a synthesized map could generate random numbers with useful statistics. The invariant density of the synthesized map has the shape of a normal (Gaussian) distribution (see Fig. 9). The transition matrix used in this example is $121 \times 121$. The points of the time-series are shown in Fig. 10, with the maximum density clearly in the mid-point of the unit-interval.

\subsection{The role of the $\beta$ parameters}

In the examples above, the values of the $\beta_{i}$ parameters were just taken to be some small value without any further explanation. If matrix $A$ represents a linear system $x_{n+1}=A x_{n}$, then the $\beta_{i}$ control how quickly the map converges to 


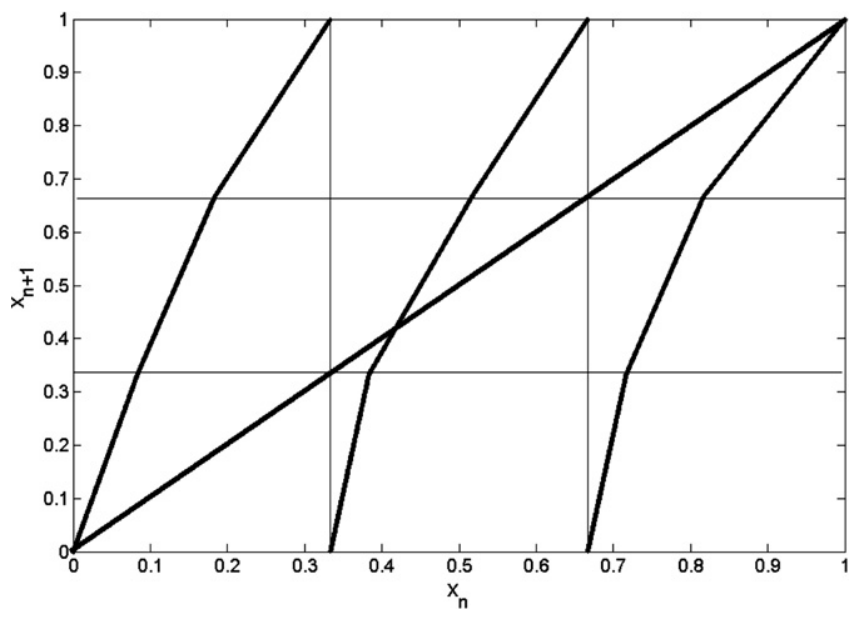

Fig. 3. One-dimensional chaotic map with partition on unit-interval shown.

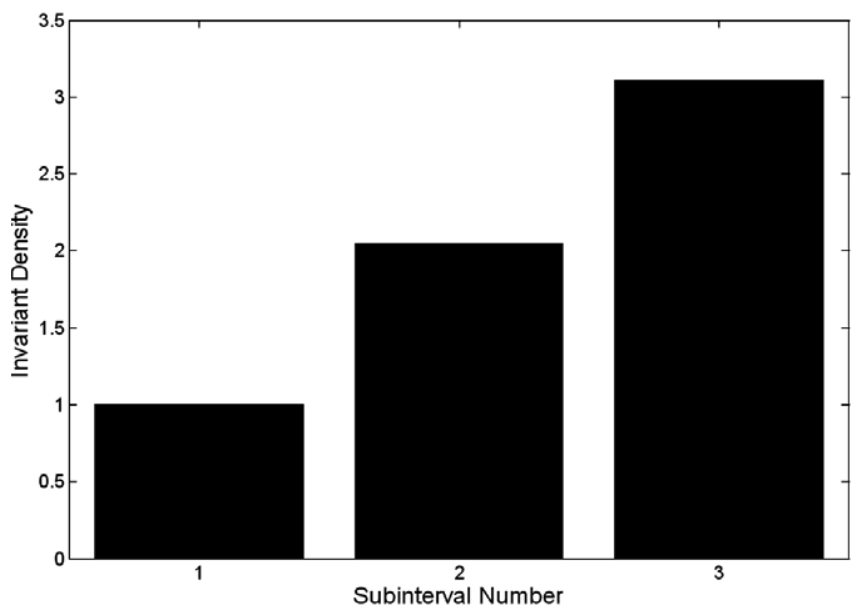

Fig. 4. Invariant density of map in Fig. 3.

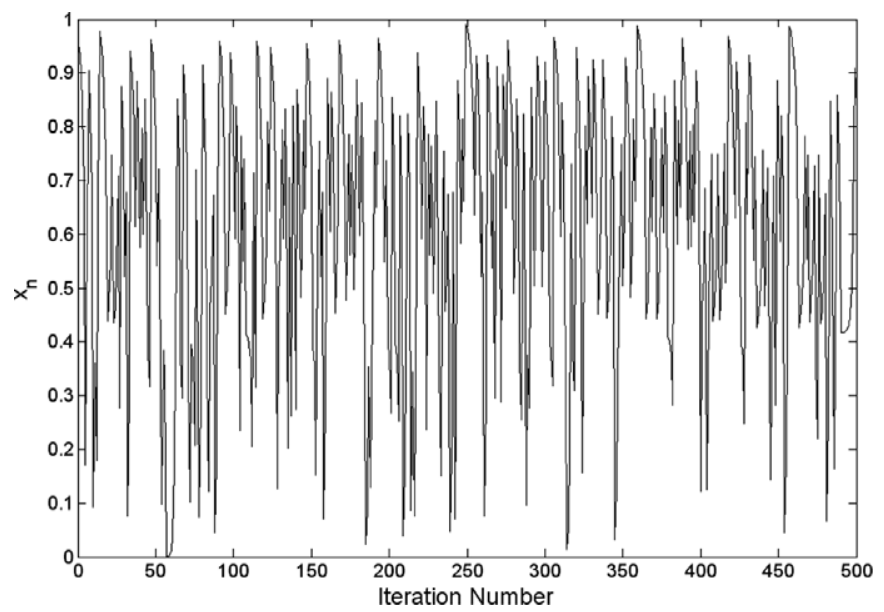

Fig. 5. Time-series of chaotic map in Fig. 3. 


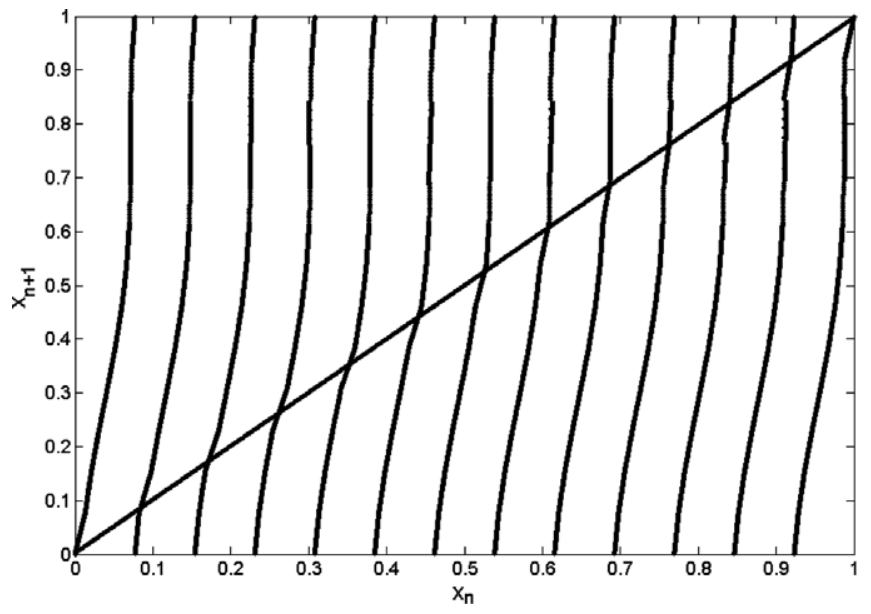

Fig. 6. One-dimensional chaotic map with sinusoidal invariant density.

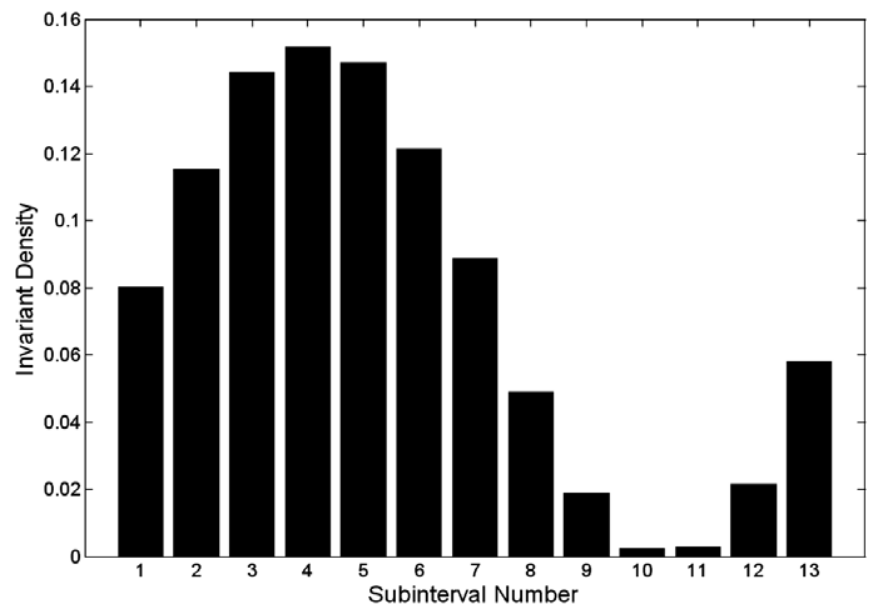

Fig. 7. Invariant density of map in Fig. 6.

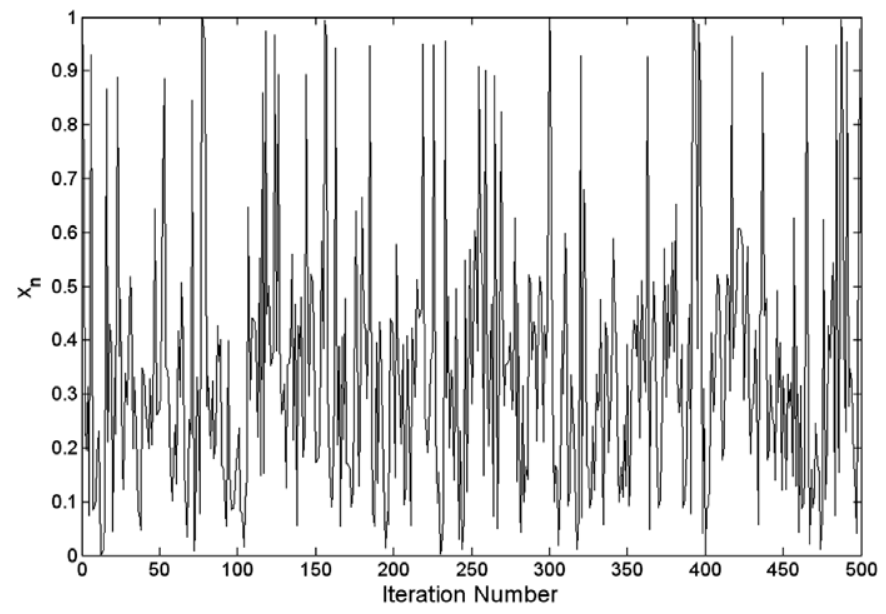

Fig. 8. Time-series of chaotic map in Fig. 6. 


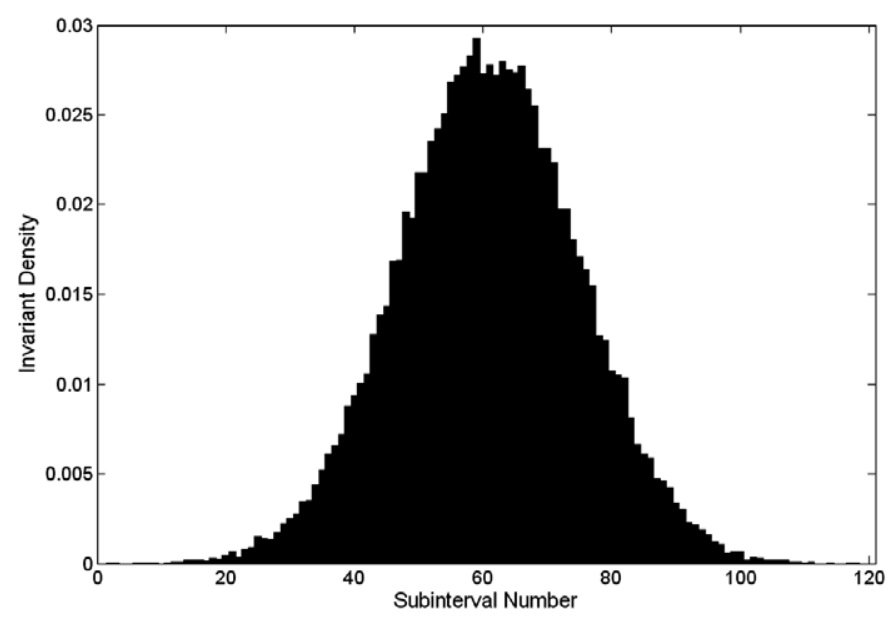

Fig. 9. Gaussian-shaped invariant density generated from a $121 \times 121$ transition matrix.

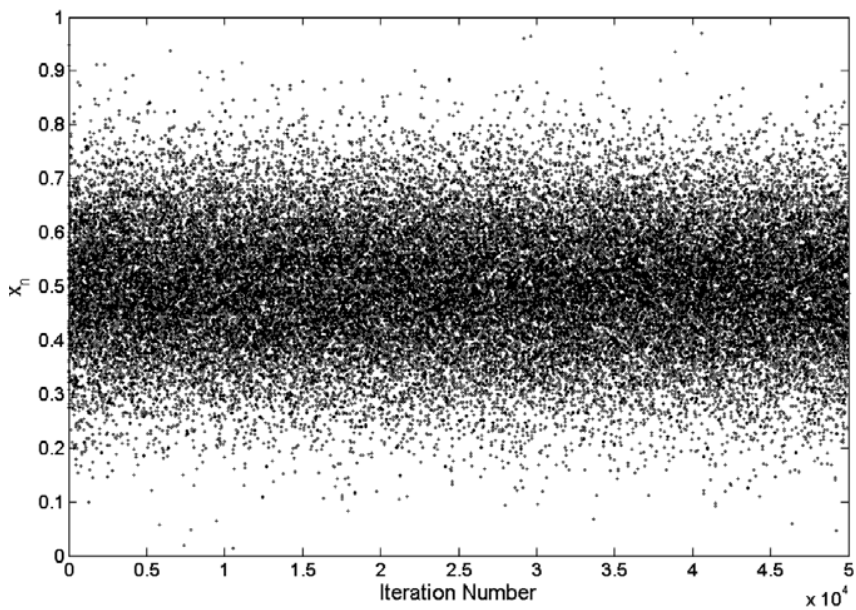

Fig. 10. Time-series of chaotic map from example (c), corresponding to invariant density in Fig. 9.

the equilibrium state upon iteration. Say we order the eigenvalues of the $A$ matrix: $\left[1, \lambda_{2}, \lambda_{3}, \ldots, \lambda_{n}\right]$, and let the corresponding normalized eigenvectors be $\left[V_{1}, V_{2}, \ldots, V_{n}\right]$. Any initial condition $x_{0}$ can be written in terms of the eigenvectors:

$$
x_{0}=\gamma_{1} V_{1}+\gamma_{2} V_{2}+\cdots+\gamma_{n} V_{n} .
$$

Thus after $k$ iterations we get

$$
A^{k} x_{0}=\gamma_{1} V_{1}+\gamma_{2} \lambda_{2}^{k} V_{2}+\cdots+\gamma_{n} \lambda_{n}^{k} V_{n} .
$$

Since the eigenvalues are interlaced with the $\beta_{i}$, and all of the $\beta_{i}<1$, it is clear from Eq. (17) that all of the terms except the first will rapidly decay to zero. The equilibrium state is thus the Perron eigenvector, as expected. The second largest eigenvalue imposes an upper bound on how fast the system approaches the equilibrium state (see Fig. 11).

The $\beta_{i}$ play a different role when matrix $A$ represents a map. Before we start iterating the map, the density is zero, and so the initial condition is at the origin. As the map is iterated, the density evolves along the Perron eigenvector. The invariant density is a statistical concept: we do not expect it to exactly equal the desired value at all times. If iterates spend long times in each subinterval before being mapped to another subinterval, then the invariant density can diverge markedly from the desired value at times. We find that it is the values of the $\beta_{i}$ that determine the length of time iterates spend in each subinterval. We now explore the mechanism giving rise to this. 


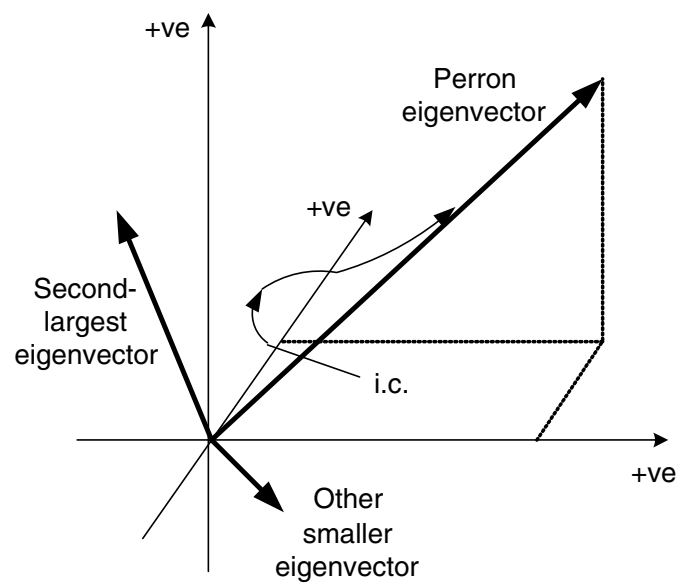

Fig. 11. Illustration of a vector evolving towards the Perron eigenvector from some initial condition. The component of the smallest eigenvector dies away first, so that the trajectory moves towards a plane spanned by the two largest eigenvectors, and eventually on to the Perron eigenvector itself.

\section{Large values of $\beta$}

If we assume that all of the $\beta_{i}$ are equal and close to 1 , then we find that the diagonal values of the $A$ matrix are approximately equal to the $\beta_{i}$, and the off-diagonal entries are all very small, due to the $1-\beta_{i}$ factors. In terms of transitions between subintervals, the probability of an iterate staying in the same subinterval is very high. Iterates are mapped to other subintervals very infrequently. Looked at dynamically, an $n \times n$ matrix will have $n$ slightly unstable fixed points, or repellors (see Fig. 13(a)). Iterates near these fixed points move away very slowly. This is also evident in the state-space plot (see Fig. 13(b)).

2. Small values of $\beta$

If the $\beta_{i}$ are small, then the transition matrix is dominated by the $\alpha_{i}$ terms. The precise values of the matrix entries are of course dependent on the desired invariant density, but it would be unusual to encounter a situation where iterates spent most of their time in one subinterval. The values of the slopes in the 1-D map tend to be large, and so the repelling fixed points are usually very unstable.

3. Various values of $\beta$

It is possible to engineer a situation where some subintervals contain weakly repelling fixed points, and others have strongly repelling fixed points, just by choosing a large or small value of $\beta$ for that subinterval.

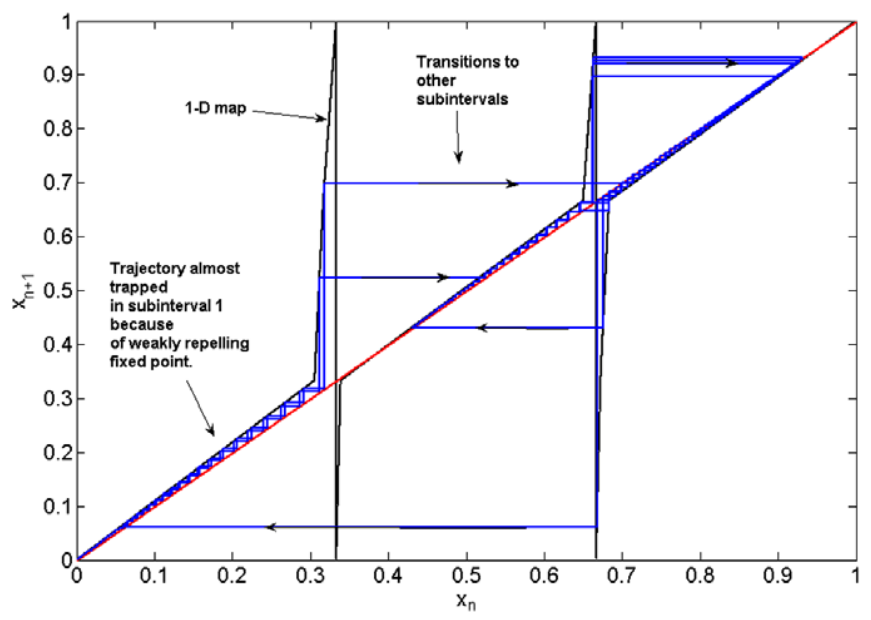

Fig. 12. Detailed plot of a 1-D map with large $\beta$ values: trajectories become trapped in subintervals and transitions occur infrequently. 
(a)

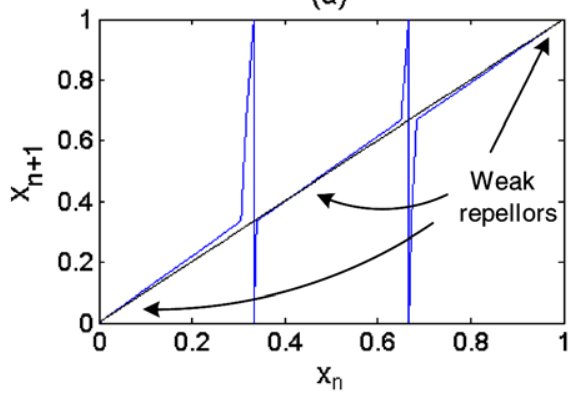

(b)

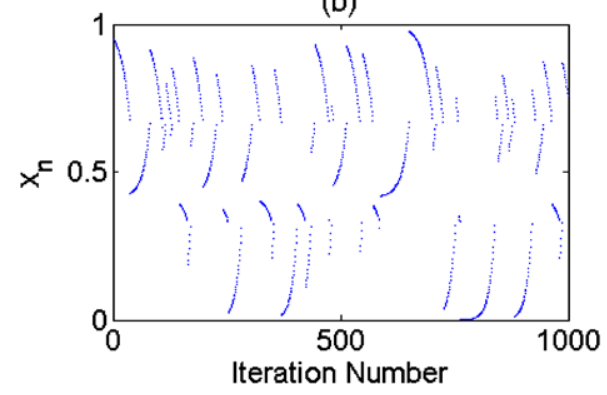

(c)

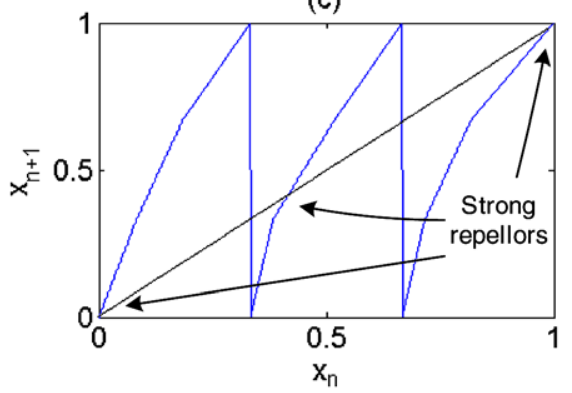

(d)

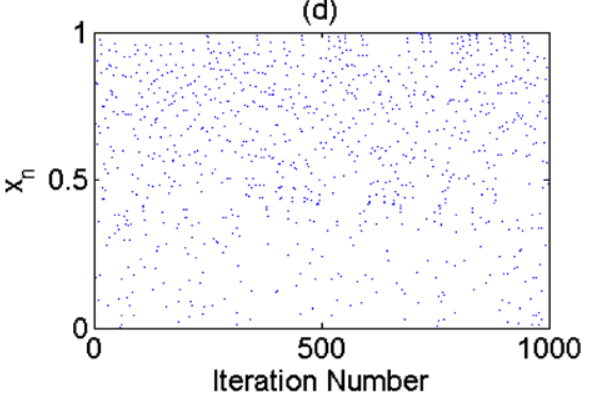

Fig. 13. 1-D map and time-series plot with: (a), (b) $\beta_{i}=0.9$; and (c), (d) $\beta_{i}=0.1$.

While the 1-D maps obtained using large values of $\beta$ still give rise to chaotic trajectories, these trajectories have long laminar regions, reminiscent of intermittency (Fig. 12). Two initially close points may remain close for a long time.

\subsection{Lyapunov exponents}

The Lyapunov exponent of a 1-D map gives the average rate of divergence of trajectories over the attractor. It is particularly straightforward to calculate for piecewise linear maps because it is related to the slopes of the map segments. For a 1-D map the Lyapunov exponent is defined as follows, using the chain rule in line 2:

$$
\lambda=\lim _{N \rightarrow \infty} \frac{1}{N} \ln \left|\frac{\mathrm{d} f^{N}\left(x_{0}\right)}{\mathrm{d} x_{0}}\right|=\lim _{N \rightarrow \infty} \frac{1}{N} \ln \left|\prod_{i=0}^{N-1} f^{\prime}\left(x_{i}\right)\right|=\lim _{N \rightarrow \infty} \frac{1}{N} \sum_{i=0}^{N-1} \ln \left|f^{\prime}\left(x_{i}\right)\right| .
$$

Essentially, it is the sum of the logs of the slope magnitudes averaged over the attractor. There are methods for computing the exponent numerically, but in this case, we know the slopes of the map, and the density of points in each subinterval, so it is possible to derive an analytic expression for the exponent. For the $2 \times 2$ case, if we assume $\beta_{1}=\beta_{2}$, then the invariant density $\rho=\left\{\alpha_{1}, \alpha_{2}\right\}$, and $\alpha_{1}+\alpha_{2}=1$. If we denote the entries of the transition matrix $a_{i j}$, then the Lyapunov exponent can be written as

$$
\lambda=\alpha_{1} a_{11} \ln \frac{1}{a_{11}}+\alpha_{1} a_{21} \ln \frac{1}{a_{21}}+\alpha_{2} a_{12} \ln \frac{1}{a_{12}}+\alpha_{2} a_{22} \ln \frac{1}{a_{22}} .
$$

This is a direct application of Eq. (18), except that the density of points in each subinterval is known a priori (given by the $\alpha_{i} a_{i j}$ terms), so we do not need to take an average over the attractor. Substituting in for the values of the $a_{i j}$, we get

$$
\begin{aligned}
\lambda= & \alpha_{1}\left\{\beta_{1}+\alpha_{1}\left(1-\beta_{1}\right) \ln \frac{1}{\beta_{1}+\alpha_{1}\left(1-\beta_{1}\right)}\right\}+\alpha_{1}\left\{\alpha_{2}\left(1-\beta_{1}\right) \ln \frac{1}{\beta_{1}+\alpha_{2}\left(1-\beta_{1}\right)}\right\} \\
& +\alpha_{2}\left\{\beta_{1}+\alpha_{2}\left(1-\beta_{1}\right) \ln \frac{1}{\beta_{1}+\alpha_{1}\left(1-\beta_{1}\right)}\right\}+\alpha_{2}\left\{\alpha_{2}\left(1-\beta_{1}\right) \ln \frac{1}{\beta_{1}+\alpha_{1}\left(1-\beta_{1}\right)}\right\} .
\end{aligned}
$$

If we let $\beta_{1} \rightarrow 0$ (a reasonable assumption), then we get a rather nice looking expression for $\lambda$ :

$$
\lambda=\alpha_{1}^{2} \ln \frac{1}{\alpha_{1}}+\alpha_{2}^{2} \ln \frac{1}{\alpha_{2}}+\alpha_{1} \alpha_{2} \ln \frac{1}{\alpha_{1} \alpha_{2}} .
$$




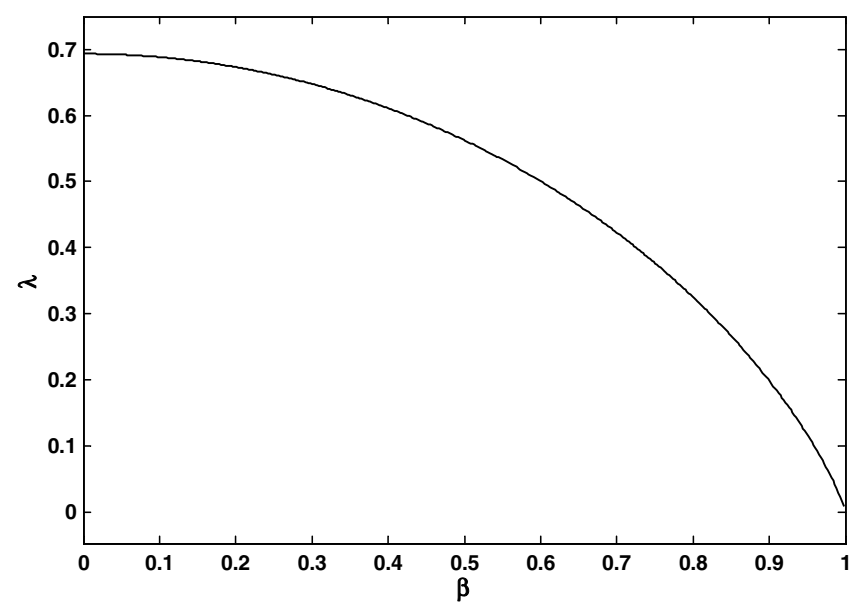

Fig. 14. Variation in Lyapunov exponent with $\beta$ and $\alpha_{1}=\alpha_{2}=0.5$.

For a $3 \times 3$ matrix, the corresponding expression is

$$
\lambda=\alpha_{1}^{2} \ln \frac{1}{\alpha_{1}}+\alpha_{2}^{2} \ln \frac{1}{\alpha_{2}}+\alpha_{3}^{2} \ln \frac{1}{\alpha_{3}}+\alpha_{1} \alpha_{2} \ln \frac{1}{\alpha_{1} \alpha_{2}}+\alpha_{2} \alpha_{3} \ln \frac{1}{\alpha_{2} \alpha_{3}}+\alpha_{3} \alpha_{1} \ln \frac{1}{\alpha_{3} \alpha_{1}} .
$$

Generalising to an $n \times n$ matrix, the Lyapunov exponent would be given by

$$
\lambda=\sum_{i=1}^{n} \alpha_{i}^{2} \ln \frac{1}{\alpha_{i}}+\sum_{i \neq j} \prod_{i, j=1}^{n} \alpha_{i} \alpha_{j} \ln \frac{1}{\alpha_{i} \alpha_{j}} .
$$

Fig. 14 shows how the Lyapunov exponent varies with $\beta$ for a $2 \times 2$ map with $\alpha_{1}=\alpha_{2}=0.5$. As expected, the maximum value of $\lambda$ occurs as $\beta \rightarrow 0$, and equals $\ln 2$.

\subsection{Nonuniform partitions}

The method may be easily modified to generate maps on nonuniform Markov partitions of the unit interval. Indeed, the synthesis method remains unchanged, as the transition matrix concerns only transitions between states. The states may be defined arbitrarily, and for one-dimensional maps, we may define the partition arbitrarily. The only difference is in the construction of the one-dimensional map, as illustrated in Fig. 15. The unit interval is partitioned into subintervals of

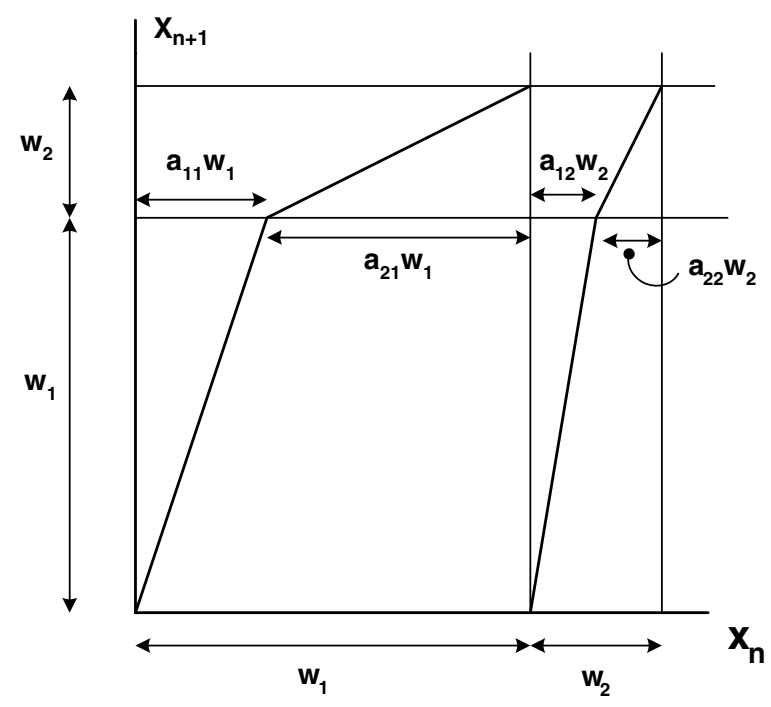

Fig. 15. Construction of 1-D map with a nonuniform partition. 
width $w_{1}, w_{2}, \ldots, w_{n}$. Each of these subintervals, $w_{i}$ is then further partitioned according to the entries of column $i$ of the transition matrix $A$. The column entries determine the widths of the piecewise linear elements of the map. For a transition from interval $i$ to interval $j$, the slope of that element of the map, $m_{j i}$, is given by

$$
m_{j i}= \pm \frac{1}{a_{j i}} \cdot \frac{w_{j}}{w_{i}} .
$$

This reduces to $m_{j i}= \pm a_{j i}^{-1}$ when the partition is uniform, as we saw previously. In Figs. 16 and 17, we show an example of the synthesis of a one-dimensional map with $\rho=[1,2,3,2,1]$ and with interval widths $w_{i}=[0.4,0.2,0.1,0.2,0.1]$. The only noteworthy effect of the nonuniform partition is that the map may no longer be everywhere expanding (i.e., some of the slopes of the map may be less than one).

\subsection{Switching between chaotic maps}

An interesting extension of the above work is to consider what happens when we switch randomly between some set of 1-D maps at each iteration of the process. This question has been considered by Boyarsky and Gora [20], where they use this process to model the famous two-slit experiment in quantum physics.

Theorem 3.1. Let $A(k) \in A_{1}, A_{2}$ and let $p_{k}=p(A(k))$ being chosen from the set. Assume the values of the $\beta_{i}$ to be the same for both maps. Let $\rho_{1}$ and $\rho_{2}$ be the invariant densities of the two maps. If we choose either $A_{1}$ or $A_{2}$ randomly (independently identically distributed) at each step of an iterative process with fixed probabilities $p_{1}$ and $p_{2}$, respectively, then the invariant density of the resultant orbit, $\rho$ is given by $\rho=p_{1} \rho_{1}+p_{2} \rho_{2}$.

Proof.

$$
\begin{aligned}
& A_{1}=\left(\begin{array}{cccc}
\beta_{1} & 0 & \cdots & 0 \\
0 & \beta_{2} & 0 & 0 \\
\vdots & 0 & \ddots & 0 \\
0 & 0 & \cdots & \beta_{n}
\end{array}\right)+\left(\begin{array}{c}
\alpha_{1} \\
\alpha_{2} \\
\vdots \\
\alpha_{n}
\end{array}\right)\left(\begin{array}{llll}
1-\beta_{1} & 1-\beta_{2} & \cdots & 1-\beta_{n}
\end{array}\right)
\end{aligned}
$$

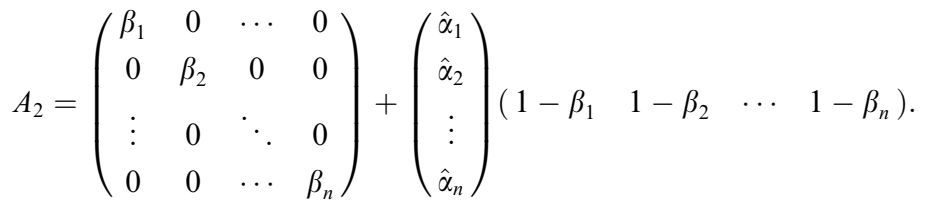

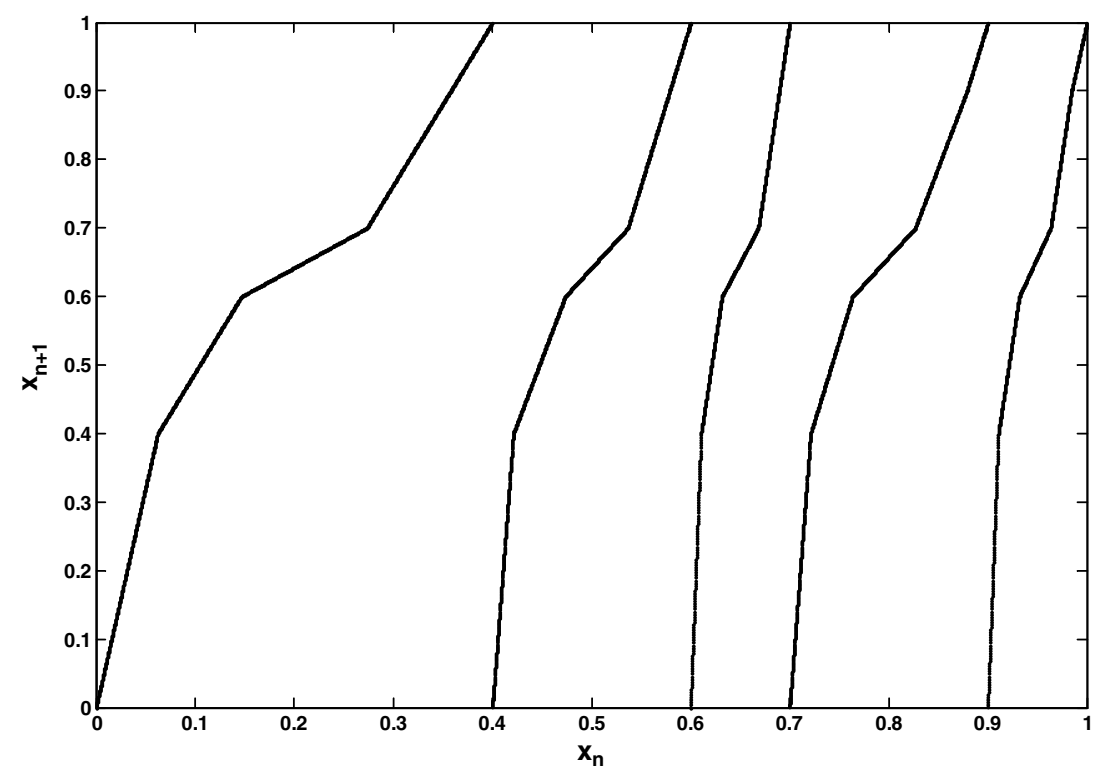

Fig. 16. Synthesized nonuniform map. 


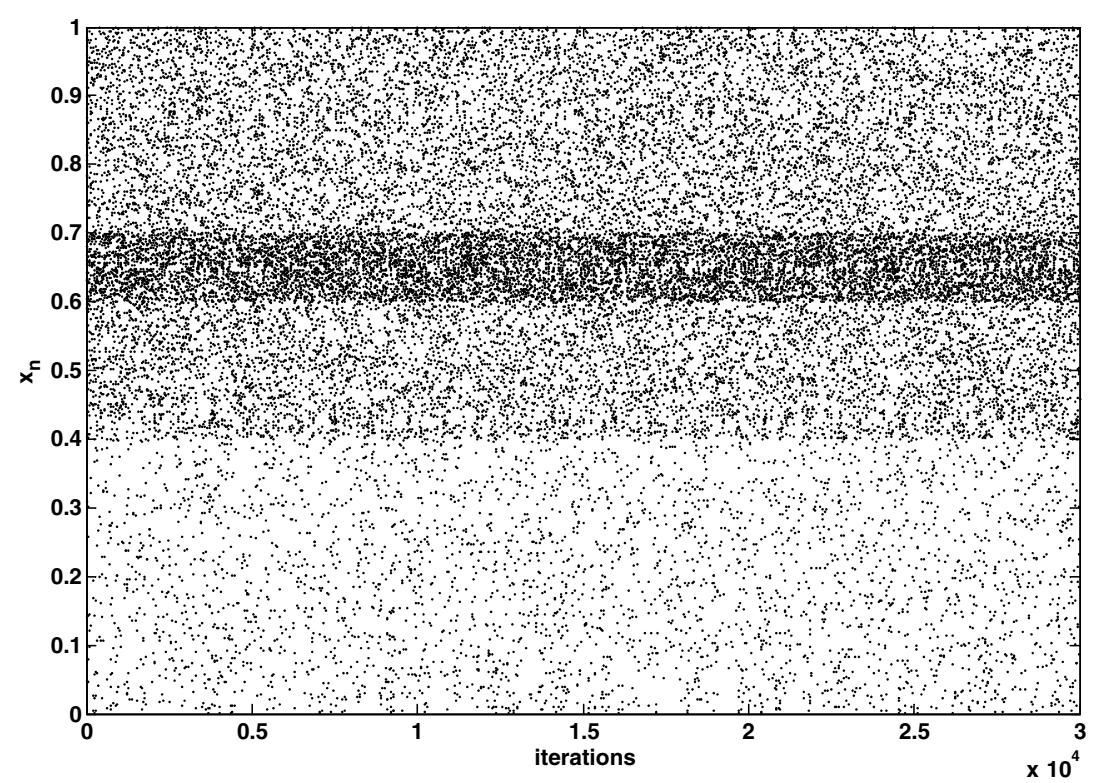

Fig. 17. Time-series of map, with different interval densities visible.

It is well-known that the expected value of the transition matrix which results when switching randomly between two matrices is given by $A_{0}=E\left(\Pi_{k}\right)=p_{1} A_{1}+p_{2} A_{2}$.

By simple substitution we can show that

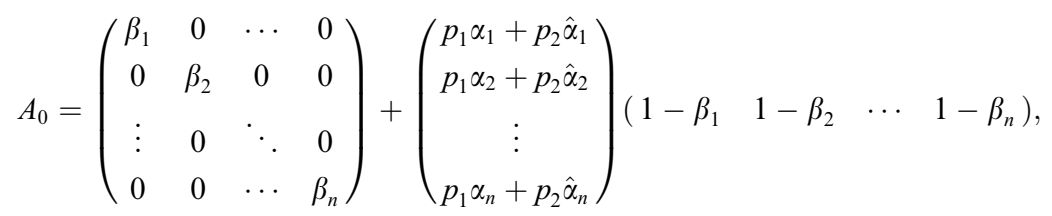

$A_{0}$ thus has a perron eigenvector of

$$
x_{\mathrm{P}}^{\mathrm{T}}=\left[\frac{p_{1} \alpha_{1}+p_{2} \hat{\alpha}_{1}}{1-\beta_{1}}, \frac{p_{1} \alpha_{2}+p_{2} \hat{\alpha}_{2}}{1-\beta_{2}}, \ldots, \frac{p_{1} \alpha_{n}+p_{2} \hat{\alpha}_{n}}{1-\beta_{n}}\right] .
$$

Theorem 3.1 shows that when the values of $\beta$ are the same in each matrix, the overall invariant density is just a weighted sum of the invariant densities of the original maps. This result also holds when switching between any number of maps. We illustrate this in the figures below where we switch between two maps. The maps have invariant densities $\rho_{1}=0.1[1,2,3,4]$ and $\rho_{2}=0.1[4,3,2,1]$, and each map is chosen with probability 0.5. As can be seen in Fig. 18 the state-space plot of the

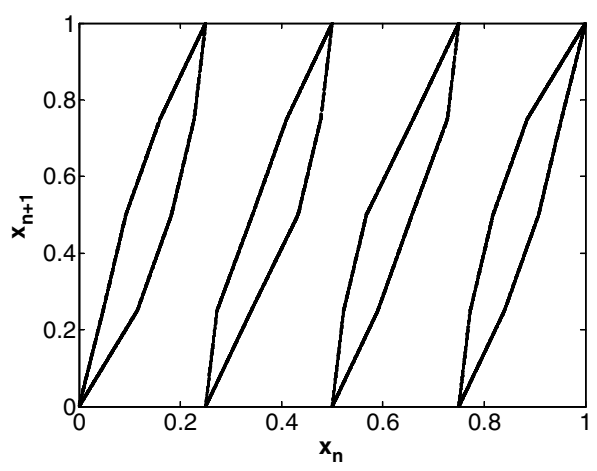

Fig. 18. State-space plot of switched-chaotic map. 


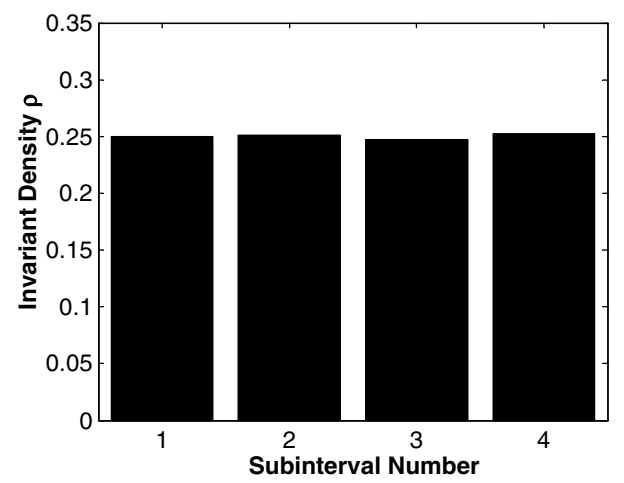

Fig. 19. Invariant density of switched chaotic map: average of constituent invariant densities.

switched system is just the superposition of the two individual maps, and the invariant density shown in Fig. 19 is the average of the two individual invariant densities.

\section{Conclusions}

The inverse Frobenius-Perron problem (IFPP) has achieved widespread attention over the past number of years as it offers a way of controlling the invariant density of chaotic maps, which in turn may lead to new applications of chaos. In this paper, we have extended our previous work on a new and simple matrix method for solving the IFPP. In the method outlined, one has complete control over the map in terms of invariant density, Lyapunov exponent, and mixing properties. The method is described is completely mechanical and highly suited to computer implementation. It could be used to generate desired distributions of (quasi-)random numbers, or to model chaotic phenomena or time-series. We also gave some preliminary results for a switched-chaotic map system.

\section{Acknowledgments}

This work was supported by the Irish Science and Technology Agency, Enterprise Ireland under research grant SC00-86. Professer Shorten was supported under grant SFI 04IN1I478.

\section{References}

[1] Lasota A, Mackey M. Chaos, fractals and noise. Springer-Verlag; 1994.

[2] Grossmann S, Thomae S. Invariant distributions and stationary autocorrelation functions of one-dimensional discrete processes. Z Naturforsch A 1977;32:1353-63.

[3] Bollt E. Controlling chaos and the inverse Frobenius-Perron problem: global stabilization of arbitrary invariant measures. Int J Bif Chaos 2000;10(5):1033-50.

[4] Gora P, Boyarsky A. A matrix solution to the inverse Frobenius-Perron problem. Proc Amer Math Soc 1993;118(2):409-14.

[5] Rogers A et al. Synthesizing chaotic maps with prescribed invariant densities. Phys Lett A 2004;330:435-41.

[6] Schuster HG. Deterministic chaos. VCH; 1989.

[7] Ott E. Chaos in dynamical systems. Cambridge University Press; 2002.

[8] Ulam S. A collection of mathematical problems. Interscience; 1960.

[9] Luenberger D. Introduction to dynamic systems. WIley; 1979.

[10] Li T. Finite approximation for the Frobenius-Perron operator: A solution to Ulam's conjecture. J Approx Theory 1976;17:177-86.

[11] Baranovsky A, Daems D. Design of one-dimensional chaotic maps with prescribed statistical properties. Int J Bif Chaos 1995;5(6):1585-98.

[12] Pingel D et al. Theory and examples of the inverse Frobenius-Perron problem for complete chaotic maps. Chaos 1999;9(2):357-66.

[13] Setti G et al. Statistical modelling of discrete-time chaotic processes - basic finite-dimensional tools and applications. Proc IEEE 2002;90(5):662-90. 
[14] Mondragon R. A model of packet traffic using a random wall model. Int J Bif Chaos 1999;9(7):1381-92.

[15] Shortenm R et al. Analysis and design of synchronised communication networks. In: Proc 12th Yale Wksp Adap Learn Sys, 2003.

[16] Berman A et al. Positive matrices associated with synchronised communication networks. Linear Algebra Appl 2004;393:47-54.

[17] Wirth F et al. Stochastic equilibria of AIMD communication networks. SIAM J Matrix Anal Appl, in press.

[18] Golub G, van Loan C. Matrix computations. Johns Hopkins University Press; 1996.

[19] Horn R, Johnson C. Matrix analysis. Cambridge University Press; 1985.

[20] Boyarsky A, Gora P. A dynamical system model for interference effects and the two-slit experiment of quantum physics. Phys Lett A $1992 ; 168: 103-12$. 